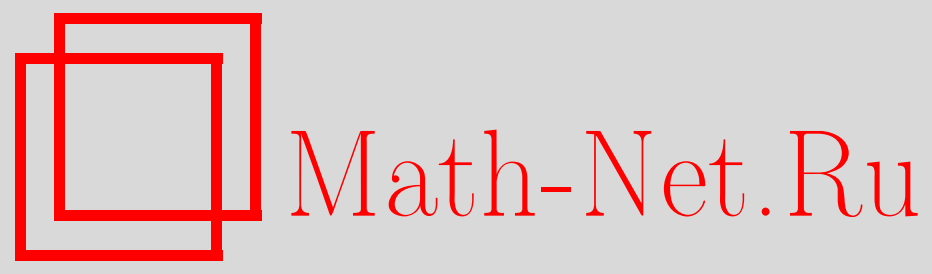

А. Н. Тимашёв, Об асимптотических разложениях для распределения числа циклов в случайной подстановке, Дискрет. матем., 2003, том 15, выпуск 3, 117-127

DOI: https://doi.org/10.4213/dm210

Использование Общероссийского математического портала Math-Net.Ru подразумевает, что вы прочитали и согласны с пользовательским соглашением http: //www.mathnet.ru/rus/agreement

Параметры загрузки:

IP : 52.205 .19 .152

26 апреля 2023 г., $17: 38: 04$

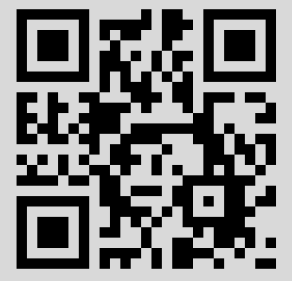


удк 519.2

\title{
Об асимптотических разложениях для распределения числа циклов в случайной подстановке
}

\author{
○ 2003 г. А. Н. Тимашёв
}

Получены явные формулы, определяющие коэффициенты асимптотических разложений в области больших уклонений для распределения числа циклов $v_{n}$ в случайной равновероятной подстановке степени $n$, то есть вероятности $\mathbf{P}\left\{v_{n}=N\right\}$ при условии, что $n, N \rightarrow \infty$ так, что

$$
1<\alpha_{0} \leqslant \alpha=\frac{n}{N} \leqslant \alpha_{1}<\infty,
$$

где $\alpha_{0}, \alpha_{1}-$ постоянные. Эти формулы выражают указанные коэффициенты через кумулянты случайной величины, имеющей распределение логарифмического ряда со специальным образом выбранным параметром. Для кумулянтов третьего и четвертого порядков приведены соответствуюшие значения. Обсуждается вопрос о точности полученных аппроксимаций. В случае, когда $n, N \rightarrow \infty$ так, что

$$
0<\gamma_{0} \leqslant \gamma=\frac{N}{\ln n} \leqslant \gamma_{1}<\infty,
$$

где $\gamma_{0}, \gamma_{1}$ - постоянные, выведены асимптотические оценки вероятностей $\mathbf{P}\left\{v_{n}=N\right\}, \mathbf{P}\left\{v_{n} \leqslant N\right\}, \mathbf{P}\left\{v_{n} \geqslant N\right\}$ с остаточным членом порядка $O\left((\ln n)^{-2}\right)$, справедливые равномерно относительно $\gamma \in\left[\gamma_{0}, \gamma_{1}\right]$. Соответствующая оценка вероятности $\mathbf{P}\left\{v_{n}=N\right\}$ улучшает ранее известные результаты для случая, когда

$$
N=\beta \ln n+o(\ln n),
$$

где $\beta$ - положительная постоянная.

Рассмотрим случайную величину $v_{n}$, равную числу циклов в подстановке, выбранной случайно равновероятно из симметрической группы степени $n$. Известно [1], что распределение этой случайной величины определяется из соотношений

$$
\mathbf{P}\left\{v_{n}=N\right\}=\frac{|s(n, N)|}{n !}, \quad N=1, \ldots, n .
$$

В равенствах (1) $s(n, N)$ - числа Стирлинга первого рода. Асимптотические оценки для вероятности $\mathbf{P}\left\{v_{n}=N\right\}$ на уровне главного члена известны при всех возможных соотношениях между параметрами $n \rightarrow \infty$ и $N=N(n)$ [2]. 
Если, в частности, $n, N \rightarrow \infty$ так, что

$$
1<\alpha_{0} \leqslant \alpha=\frac{n}{N} \leqslant \alpha_{1}<\infty,
$$

где $\alpha_{0}, \alpha_{1}$ - постоянные, то соответствуюшая формула для главного члена впервые получена в [3] и имеет следующий вид: равномерно относительно $\alpha \in\left[\alpha_{0}, \alpha_{1}\right]$

$$
|s(n, N)|=\frac{n !}{N !} \frac{\left(-\ln \left(1-x_{0}\right)\right)^{N}}{x_{0}^{n}} \frac{1}{\sqrt{2 \pi N \sigma_{0}^{2}}}(1+O(1 / N)),
$$

где $x=x_{0}-$ единственный принадлежащий интервалу $(0,1)$ корень уравнения

$$
\begin{aligned}
& \frac{x}{(x-1) \ln (1-x)}=\alpha, \\
& \sigma_{0}^{2}=\frac{\alpha\left(\alpha x_{0}-\alpha+1\right)}{1-x_{0}}>0 .
\end{aligned}
$$

В работе автора [4] для чисел $|s(n, N)|$ при условиях (2) и $n, N \rightarrow \infty$ получено асимптотическое разложение вида (3), справедливое равномерно относительно $\alpha \in\left[\alpha_{0}, \alpha_{1}\right]$, в котором остаточный член $O(1 / N)$ заменен на асимптотический степенной ряд $\sum_{m=1}^{\infty} A_{m} N^{-m}$. Для коэффициентов $A_{m}$ в [4] приведены имеющие достаточно сложный вид нелинейные рекуррентные соотношения с известными начальными условиями. Таким образом, при $n, N \rightarrow \infty$ и при выполнении условий (2) из (1) и (3) получаем, что равномерно относительно $\alpha \in\left[\alpha_{0}, \alpha_{1}\right]$ справедливо асимптотическое разложение

$$
\mathbf{P}\left\{v_{n}=N\right\} \sim \frac{\left(-\ln \left(1-x_{0}\right)\right)^{N}}{N ! x_{0}^{n}} \frac{1}{\sqrt{2 \pi N \sigma_{0}^{2}}}\left(1+\sum_{m=1}^{\infty} A_{m} N^{-m}\right) .
$$

Поскольку при $n \rightarrow \infty$ (см. [5])

$$
\mathbf{E} v_{n}=\ln n(1+o(1)),
$$

из соотношений (1) и (2) следует, что разложение (6) определяет асимптотику вероятностей $\mathbf{P}\left\{v_{n}=N\right\}$ в области больших уклонений случайной величины $v_{n}$. Тем не менее, локальная нормальная теорема для $v_{n}$ [6], а также другие известные оценки вероятностей $\mathbf{P}\left\{v_{n}=N\right\}$ (см. [2]) при их практическом применении дают во многих случаях худший результат по сравнению с оценкой (6) (что будет показано ниже на примерах).

В работе получены явные формулы для коэффициентов $A_{m}, m=1,2 \ldots$ Эти формулы выражают коэффициенты $A_{m}$ через кумулянты случайной величины $\xi_{1}$, имеющей распределение логарифмического ряда с параметром $x$ [6], то есть такой, что

$$
\mathbf{P}\left\{\xi_{1}=k\right\}=-\frac{x^{k}}{k \ln (1-x)}, \quad k=1,2, \ldots, \quad x \in(0,1) .
$$

Вывод получаемых ниже соотношений основан на применении известных равенств [6]

$$
\mathbf{P}\left\{v_{n}=N\right\}=\frac{(-\ln (1-x))^{N}}{N ! x^{n}} \mathbf{P}\left\{\xi_{1}+\ldots+\xi_{N}=n\right\}, \quad N=1, \ldots, n .
$$

В равенствах (8) случайные величины $\xi_{1}, \ldots, \xi_{N}$ независимы и одинаково распределены. 
Теорема 1. Пусть $n, N \rightarrow \infty$ так, что выполнены условия (2). Тогда равномерно относительно $\alpha \in\left[\alpha_{0}, \alpha_{1}\right]$ справедливо асимптотическое разложение (6), в котором величины $x_{0}$ и $\sigma_{0}^{2}$ определяются равенствами (4), (5), а коэффициенты $A_{m}$ находятся из соотношений

$$
A_{m}=\sum_{k_{1}+2 k_{2}+3 k_{3}+\ldots+2 m k_{2 m}=2 m} \frac{(-1)^{l}(2 l) !}{l ! 2^{l}} \prod_{s=1}^{2 m} \frac{1}{\left(k_{s}\right) !}\left(\frac{\varkappa_{s+2}}{(s+2) ! \sigma_{0}^{s+2}}\right)^{k_{s}}
$$

где $m=1,2, \ldots$ и суммирование в (9) проводится по всевозможным упорядоченным наборам неотричательных чельх чисел $k_{1}, \ldots, k_{2 m}$, удовлетворяюших условию $k_{1}+2 k_{2}+3 k_{3}+\ldots+2 m k_{2 m}=2 m$. При этом $l=m+k_{1}+\ldots+k_{2 m} u x_{s}-к у м у-$ лянт порядка s случайной величины $\xi_{1}$, имеющей распределение вида (7) при $x=x_{0}$, $s=3,4, \ldots$

Доказательство. Используем равенства (7) и (8), полагая $x=x_{0}$. Как нетрудно подсчитать, согласно (2) и (4)

$$
\mathbf{E} \xi_{1}=\frac{x_{0}}{\left(x_{0}-1\right) \ln \left(1-x_{0}\right)}=\frac{n}{N}=\alpha
$$

поэтому

$$
\mathbf{P}\left\{\xi_{1}+\ldots+\xi_{N}=n\right\}=\frac{1}{\sqrt{2 \pi N D \xi_{1}}}(1+O(1 / N)),
$$

где $\mathbf{D} \xi_{1}=\sigma_{0}^{2}>0$.

Чтобы уточнить формулу (10), оценим коэффициенты ряда Крамера-Эссеена, дающего асимптотическое разложение остаточного члена вида $O(1 / N)$ в этой формуле. Так как $\mathbf{E}\left(\xi_{1}+\ldots+\xi_{N}\right)=n$ при $x=x_{0}$, указанные коэффициенты $A_{m}$ могут быть отличны от нуля только при целых отрицательных степенях вида $N^{-m}, m=1,2, \ldots$ Известно [7], что эти коэффициенты выражаются через кумулянты $\varkappa_{s}$ порядка $s, s=3,4, \ldots$, случайной величины $\xi_{1}$. Точнее, при $m=1,2, \ldots$ согласно [7] можно утверждать, что справедливы формулы (9). Таким образом, при условиях теоремы 1 равномерно относительно $\alpha \in\left[\alpha_{0}, \alpha_{1}\right]$ (см. аналогичное утверждение в статье автора [16])

$$
\mathbf{P}\left\{\xi_{1}+\ldots+\xi_{N}=n\right\} \sim \frac{1}{\sqrt{2 \pi N \sigma_{0}^{2}}}\left(1+\sum_{m=1}^{\infty} A_{m} N^{-m}\right) .
$$

Подставляя разложение (11) в (8), при $x=x_{0}$ получаем (6). Теорема 1 доказана.

Следствие 1. При условиях теоремы 1 равномерно относительно $\alpha \in\left[\alpha_{0}, \alpha_{1}\right]$

$$
\begin{aligned}
\mathbf{P}\left\{v_{n}=N\right\} & =\frac{\left(-\ln \left(1-x_{0}\right)\right)^{N}}{N ! x_{0}^{n}} \frac{1}{\sqrt{2 \pi N \sigma_{0}^{2}}}\left(1+A_{1} N^{-1}+O\left(N^{-2}\right)\right), \\
A_{1} & =\frac{\varkappa_{4}}{8 \sigma_{0}^{4}}-\frac{5 \varkappa_{3}^{2}}{24 \sigma_{0}^{6}}, \\
\varkappa_{3} & =\frac{\alpha\left(1+x_{0}\right)}{\left(1-x_{0}\right)^{2}}-3 \alpha \sigma_{0}^{2}-\alpha^{3}, \\
\varkappa_{4} & =\frac{\alpha\left(1-4 \alpha+4 x_{0}+(1+4 \alpha) x_{0}^{2}\right)}{\left(1-x_{0}\right)^{3}}+6 \alpha^{2} \sigma_{0}^{2}+3 \alpha^{4}-3 \sigma_{0}^{4} .
\end{aligned}
$$


Доказательство. Заметим, что разложение (6) следует понимать в том смысле, что при условиях теоремы 1 для любого фиксированного значения $M=1,2, \ldots$ равномерно относительно $\alpha \in\left[\alpha_{0}, \alpha_{1}\right]$ справедливо равенство

$$
\mathbf{P}\left\{v_{n}=N\right\}=\frac{\left(-\ln \left(1-x_{0}\right)\right)^{N}}{N ! x_{0}^{n}} \frac{1}{\sqrt{2 \pi N \sigma_{0}^{2}}}\left(1+\sum_{m=1}^{M} A_{m} N^{-m}+O\left(N^{-(M+1)}\right)\right) .
$$

В частности, при $M=1$ из (16) следует (12). Равенство (13) для коэффициента $A_{1}$ следует из (9) при $m=1$. Полагая в (7) $x=x_{0}$, нетрудно проверить, что

$$
\begin{aligned}
\mathbf{E} \xi_{1}^{3} & =\frac{\alpha\left(1+x_{0}\right)}{\left(1-x_{0}\right)^{2}} \\
\mathbf{E} \xi_{1}^{4} & =\frac{\alpha\left(1+4 x_{0}+x_{0}^{2}\right)}{\left(1-x_{0}\right)^{3}}
\end{aligned}
$$

Кроме того,

$$
\begin{aligned}
& \varkappa_{3}=\mathbf{E}\left(\xi_{1}-\alpha\right)^{3}=\mathbf{E} \xi_{1}^{3}-3 \alpha \sigma_{0}^{2}-\alpha^{3} \\
& \varkappa_{4}=\mathbf{E}\left(\xi_{1}-\alpha\right)^{4}-3 \sigma_{0}^{4} .
\end{aligned}
$$

Из (17)-(20) получаем соотношения (14), (15). Следствие 1 доказано.

Замечание 1. Оценка (12) обладает высокой точностью приближения даже при сравнительно небольших значениях $n$ и $N$. Так, например, если $n=12$ и $N=8$, то $\alpha=3 / 2$, $x_{0} \approx 0,5335893$ с точностью до $10^{-7}$ и главный член в (12) равен 0,0007650 (с той же точностью). Первое приближение, полученное из (12) путем отбрасывания остаточного члена порядка $O\left(N^{-2}\right)$, дает значение 0,0007457 , в то время как

$$
\mathbf{P}\left\{v_{12}=8\right\} \approx 0,0007462 .
$$

Если $n=20$ и $N=3$, то $\alpha=20 / 3, x_{0} \approx 0,94989$ с точностью $10^{-5}$, в этом случае оценки, полученные из (12), дают значения 0,30589 и 0,28682 , в то время как

$$
\mathbf{P}\left\{v_{20}=3\right\} \approx 0,27482
$$

(с той же точностью).

Приближения, полученные по локальной нормальной теореме и формуле Жордана [8], дают менее точные значения, равные соответственно 0,23049 и 0,31915.

Замечание 2. Формулы для коэффициентов $A_{m}$, аналогичные (13)-(15), можно выписать и при $m \geqslant 2$. Так, например, при $m=2$ из (9) получаем, что

$$
A_{2}=-\frac{\varkappa_{6}}{48 \sigma_{0}^{6}}+\frac{56 \varkappa_{3} \varkappa_{5}+35 \varkappa_{4}^{2}}{384 \sigma_{0}^{8}}-\frac{35 \varkappa_{3}^{2} \varkappa_{4}}{64 \sigma_{0}^{10}}+\frac{385 \varkappa_{3}^{4}}{1152 \sigma_{0}^{12}} \text {. }
$$

Замечание 3. При условиях (2) и $n, N \rightarrow \infty$ асимптотические разложения для чисел Стирлинга второго рода, аналогичные (6), получены автором в [4]. Формулы для коэффициентов этих разложений, соответствующие (9), приведены в [9]. 
Рассмотрим теперь другую область изменения параметров $n, N$, когда $n, N \rightarrow \infty$ так, что

$$
0<\gamma_{0} \leqslant \gamma=\frac{N}{\ln n} \leqslant \gamma_{1}<\infty
$$

где $\gamma_{0}, \gamma_{1}$ - постоянные.

Известно [2], что если при $n \rightarrow \infty, N=\beta \ln n+o(\ln n)$, где $\beta$ - положительная постоянная, то

$$
\mathbf{P}\left\{v_{n}=N\right\}=\frac{(\ln n)^{N}}{N ! n \Gamma(\beta)}(1+o(1)) .
$$

Обобщая (22) на случай, когда выполнены условия (21), докажем следующее общее утверждение.

Теорема 2. Пусть $n, N \rightarrow \infty$ так, что справедливы условия (21). Тогда равномерно относительно $\gamma \in\left[\gamma_{0}, \gamma_{1}\right]$

$$
\mathbf{P}\left\{v_{n}=N\right\}=\frac{(\ln n)^{N}}{N ! n \Gamma(\gamma)}\left(1+\frac{\gamma\left(\psi^{\prime}(\gamma)-(\psi(\gamma))^{2}\right)}{2 \ln n}+O\left(1 /(\ln n)^{2}\right)\right),
$$

гдe

$$
\psi\left(\gamma=\frac{\Gamma^{\prime}(\gamma)}{\Gamma(\gamma)}\right)=(\ln \Gamma(\gamma))^{\prime}
$$

Доказательство. Для обоснования равенства (23) используем метод перевала, исходя из интегрального представления [8]

$$
\mathbf{P}\left\{v_{n}=N\right\}=\frac{1}{2 \pi i n !} \oint \frac{(z+1) \ldots(z+n-1)}{z^{N}} d z .
$$

Интегрирование в (24) осуществляется по окружности $|z|=z_{0}>0$, где значение $z_{0}$ будет выбрано позднее. Формулу (24) можно представить в виде

$$
\mathbf{P}\left\{v_{n}=N\right\}=\frac{1}{2 \pi i n !} \oint z^{-1} \exp (N \omega(z)) d z,
$$

где

$$
\omega(z)=\frac{1}{N} \sum_{k=0}^{n-1} \ln (z+k)-\ln z
$$

и для логарифма выбирается та непрерывная ветвь, которая принимает вещественные значения на вещественной положительной полуоси.

Далее,

$$
\omega^{\prime}(z)=\frac{1}{N} \sum_{k=0}^{n-1} \frac{1}{z+k}-\frac{1}{z}=0,
$$

если

$$
f(z)=\sum_{k=0}^{n-1} \frac{z}{z+k}=N
$$


При фиксированном $n$ функция $f(z)$ непрерывна и строго возрастает от 1 до $n$ при возрастании $z$ от 0 до $\infty$, поэтому из (21) следует, что уравнение (27) для заданных достаточно больших значений $n$ и $N$ имеет единственный положительный корень $z_{0}=z_{0}(n, N)$. Из (26) и (27) получаем, что

$$
\omega^{\prime \prime}\left(z_{0}\right)=-\frac{1}{N} \sum_{k=0}^{n-1} \frac{1}{\left(z_{0}+k\right)^{2}}+\frac{1}{z_{0}^{2}}>0
$$

при $n \geqslant 2$, и поэтому $z_{0}$ - точка перевала первого порядка функции $\omega(z)$. Но тогда согласно [10] из (25) следует, что при условиях (21) и $n \rightarrow \infty$ равномерно относительно $\gamma \in\left[\gamma_{0}, \gamma_{1}\right]$

$$
\mathbf{P}\left\{v_{n}=N\right\}=\frac{z_{0}^{-1} \exp \left(N \omega\left(z_{0}\right)\right)}{n ! \sqrt{2 \pi N \omega^{\prime \prime}\left(z_{0}\right)}}\left(1+\frac{B}{N}+O\left(\frac{1}{N^{2}}\right)\right) .
$$

где (см. [11])

$$
B=-\frac{1}{4 \omega^{\prime \prime}\left(z_{0}\right)}\left(\frac{4}{z_{0}^{2}}+\frac{2 \omega^{\prime \prime \prime}\left(z_{0}\right)}{z_{0} \omega^{\prime \prime}\left(z_{0}\right)}+\frac{5\left(\omega^{\prime \prime \prime}\left(z_{0}\right)\right)^{2}}{6\left(\omega^{\prime \prime}\left(z_{0}\right)\right)^{2}}-\frac{\omega^{(4)}\left(z_{0}\right)}{2 \omega^{\prime \prime}\left(z_{0}\right)}\right) .
$$

Известно [12], что при $z>0$

$$
\begin{aligned}
& \psi(z)=\lim _{n \rightarrow \infty}\left(\ln n-\sum_{k=0}^{n-1} \frac{1}{z+k}\right), \\
& \psi^{\prime}(z)=\sum_{k=0}^{\infty} \frac{1}{(z+k)^{2}} .
\end{aligned}
$$

Используя (21), из (27) получаем, что при $z=z_{0}$

$$
z \sum_{k=0}^{n-1} \frac{1}{z+k}=\gamma \ln n
$$

Из равенств (30)-(32) нетрудно получить, что при $n \rightarrow \infty$ равномерно относительно $\gamma \in\left[\gamma_{0}, \gamma_{1}\right]$ (промежуточные выкладки мы опускаем)

$$
z_{0}=\gamma+\frac{\gamma \psi(\gamma)}{\ln n}+\frac{\gamma \psi(\gamma)\left(\psi(\gamma)+\gamma \psi^{\prime}(\gamma)\right)}{(\ln n)^{2}}+O\left(\frac{1}{(\ln n)^{3}}\right) .
$$

Подставляя оценку (33) в формулу для $\omega^{\prime \prime}\left(z_{0}\right)$, приведенную выше, и осушествляя элементарные преобразования, выводим, что

$$
\left.\omega^{\prime \prime} z_{0}\right)=z_{0}^{-2}\left(1-\frac{\gamma \psi^{\prime}(\gamma)}{\ln n}+O\left(\frac{1}{(\ln n)^{2}}\right)\right) .
$$

Следовательно,

$$
\frac{z_{0}^{-1}}{\sqrt{\omega^{\prime \prime}\left(z_{0}\right)}}=1+\frac{\gamma \psi^{\prime}(\gamma)}{2 \ln n}+O\left(\frac{1}{(\ln n)^{2}}\right)
$$


Из соотношения

$$
\exp \left(N \omega\left(z_{0}\right)\right)=\frac{\Gamma\left(z_{0}+n\right)}{\Gamma\left(z_{0}\right) z_{0}^{N}}
$$

и формулы Стирлинга для гамма-функции получаем, что

$$
\frac{\exp \left(N \omega\left(z_{0}\right)\right)}{n !}=\frac{n^{z_{0}-1-\gamma \ln z_{0}}}{\Gamma\left(z_{0}\right)}(1+O(1 / n)) .
$$

Согласно (33)

$$
\Gamma\left(z_{0}\right)=\Gamma(\gamma)\left(1+\frac{\gamma(\psi(\gamma))^{2}}{\ln n}+O\left(\frac{1}{(\ln n)^{2}}\right)\right)
$$

поэтому

$$
\frac{\exp \left(N \omega\left(z_{0}\right)\right)}{n !}=\frac{n^{z_{0}-1-\gamma \ln z_{0}}}{\Gamma(\gamma)}\left(1-\frac{\gamma(\psi(\gamma))^{2}}{\ln n}+O\left(\frac{1}{(\ln n)^{2}}\right)\right),
$$

и с учетом (34)

$$
\frac{z_{0}^{-1} \exp \left(N \omega\left(z_{0}\right)\right)}{n ! \sqrt{\omega^{\prime \prime}\left(z_{0}\right)}}=\frac{n^{z_{0}-1-\gamma \ln z_{0}}}{\Gamma(\gamma)}\left(1+\frac{\gamma\left(\psi^{\prime}(\gamma)-2(\psi(\gamma))^{2}\right)}{2 \ln n}+O\left(\frac{1}{(\ln n)^{2}}\right)\right) .
$$

Использование формулы Стирлинга для $N$ ! и условий (21) приводит к равенству

$$
\frac{1}{\sqrt{2 \pi N}}=\frac{(\ln n)^{N}}{N !} n^{\gamma(\ln \gamma-1)}\left(1+\frac{1}{12 \gamma \ln n}+O\left(\frac{1}{(\ln n)^{2}}\right)\right) .
$$

Таким образом, согласно (35) и (36)

$$
\begin{aligned}
\frac{z_{0}^{-1} \exp \left(N \omega\left(z_{0}\right)\right)}{n ! \sqrt{2 \pi N \omega^{\prime \prime}\left(z_{0}\right)}}= & \frac{(\ln n)^{N} n^{z_{0}-\gamma-\gamma \ln z_{0}+\gamma \ln \gamma}}{N ! n \Gamma(\gamma)} \\
& \times\left(1+\frac{\gamma\left(\psi^{\prime}(\gamma)-2(\psi(\gamma))^{2}\right)+(1 / 6) \gamma^{-1}}{2 \ln n}+O\left(\frac{1}{(\ln n)^{2}}\right)\right) .
\end{aligned}
$$

Из (29) и (33) с учетом легко проверяемых соотношений

$$
\begin{aligned}
\omega^{\prime \prime}\left(z_{0}\right) & =\gamma^{-2}(1+O(1 /(\ln n))), \\
\omega^{\prime \prime \prime}\left(z_{0}\right) & =-2 \gamma^{-3}(1+O(1 /(\ln n))), \\
\omega^{(4)}\left(z_{0}\right) & =6 \gamma^{-4}(1+O(1 /(\ln n)))
\end{aligned}
$$

следует, что

$$
B=-\frac{1}{12}+O(1 /(\ln n))
$$

Далее, согласно (33)

$$
n^{z_{0}-\gamma-\gamma \ln z_{0}+\gamma \ln \gamma}=1+\frac{\gamma(\psi(\gamma))^{2}}{2 \ln n}+O\left(\frac{1}{(\ln n)^{2}}\right) .
$$

Подставляя оценки (37)-(39) в (28), получаем (23). Поскольку эти оценки равномерны по $\gamma \in\left[\gamma_{0}, \gamma_{1}\right]$, то теорема 2 доказана. 
Замечание 4. При $n \rightarrow \infty$ и $N=\beta \ln n+o(\ln n)$, где $\beta$ - положительная постоянная, оценка скорости сходимости в (22) была получена в [13]. Для случая $\beta=1$ соответствующая оценка приведена в [14], более точная оценка доказана в [15], а именно, если $n \rightarrow \infty, N=\ln n+x \sqrt{\ln n}$, где $x=x(n)=o(\sqrt{\ln n})$, то

$$
\mathbf{P}\left\{v_{n}=N\right\}=\frac{(\ln n)^{N}}{N ! n}\left(1+O\left(\frac{|x|}{\sqrt{\ln n}}+\frac{1}{\ln n}\right)\right) .
$$

Формула (40) следует из (23), поскольку согласно (21) $\gamma=1+x / \sqrt{\ln n}$, и поэтому $\Gamma(\gamma)=1+O(x / \sqrt{\ln n})$.

Существенным усилением (40) является следующее утверждение.

Следствие 2. Пусть $n \rightarrow \infty, N=\ln n+x \sqrt{\ln n}$, где $x=x(n)=o(\sqrt{\ln n})$. Тогда

$$
\begin{aligned}
& \mathbf{P}\left\{v_{n}=N\right\}=\frac{(\ln n)^{N}}{N ! n}\left(1+C x / \sqrt{\ln n}+\left(\pi^{2} / 12-C^{2} / 2-\left(\pi^{2} / 6+C^{2}\right) x^{2}\right)(\ln n)^{-1}\right. \\
&+\left(\left(\pi^{2} / 12\right)(1+3 C)-C^{2}(C+1) / 2-\xi(3)\right) x \\
&\left.\left.+\left(C \pi^{2} / 2+C^{3}+2 \xi(3)\right) x^{3}\right)(\ln n)^{-3 / 2}+O\left(\left(1+x^{4}\right) /(\ln n)^{2}\right)\right),
\end{aligned}
$$

где $C=0,577215 \ldots$ - постоянная Эйлера,

$$
\xi(3)=\sum_{k=1}^{\infty} \frac{1}{k^{3}}=1,2020569 \ldots
$$

Оценка (41) следует из (23), если использовать известные равенства

$$
\psi(1)=-C, \quad \psi^{\prime}(1)=\pi^{2} / 6, \quad \psi^{\prime \prime}(1)=-2 \xi(3), \quad \psi^{\prime \prime \prime}(1)=\pi^{4} / 15
$$

и учесть, что $\psi(\gamma)=\psi(1+x / \sqrt{\ln n})$.

Замечание 5. Если, в частности, величина $x=x(n)$ ограничена, то равномерно относительно целых положительных значений $N=\ln n+x \sqrt{\ln n}$, для которых $|x| \leqslant c$, где $c-$ постоянная (см. [2])

$$
\mathbf{P}\left\{v_{n}=N\right\}=\frac{1}{\sqrt{2 \pi \ln n}} e^{-x^{2} / 2}(1+o(1)) .
$$

Уточняя (42), можно получить следующее утверждение.

Следствие 3. При $n \rightarrow \infty u N=\ln n+x \sqrt{\ln n}$ равномерно относительно цельх положительных значений $N$, для которых $|x| \leqslant c$, где $c-$ постоянная,

$$
\begin{aligned}
\mathbf{P}\left\{v_{n}\right. & =N\}=\frac{1}{\sqrt{2 \pi \ln n}} e^{-x^{2} / 2} \\
& \times\left(1+\frac{(C-1 / 2) x+x^{3} / 6}{\sqrt{\ln n}}+A(x)(\ln n)^{-1}+B(x)(\ln n)^{-3 / 2}+O\left(1 /(\ln n)^{2}\right)\right),
\end{aligned}
$$


где

$$
\begin{aligned}
A(x)= & \pi^{2} / 12-C^{2} / 2-1 / 12-\left(\pi^{2} / 6+C^{2}+C / 2-1 / 8\right) x^{2}+((C-1) / 6) x^{4}-x^{6} / 72 . \\
B(x)=\left(\pi^{2} / 24+\pi^{2} C / 4-C^{3} / 2-C^{2} / 4-C / 12-\xi(3)+1 / 8\right) x & \\
& +\left(7 \pi^{2} / 72+\pi^{2} C / 2+C^{3}+(5 / 12) C^{2}+C / 8+2 \xi(3)-11 / 144\right) x^{3} \\
& \quad-\left(\pi^{2} / 36+C^{2} / 6+C / 6-9 / 80\right) x^{5}-(C / 72-1 / 48) x^{7}+x^{9} / 1296
\end{aligned}
$$

и С и $\xi(3)$ определены в следствии 2.

Обоснование формулы (43) проводится с использованием стандартной техники асимптотических оценок, исходя из (41), применением формулы Стирлинга.

Замечание 6. Нетрудно убедиться в том, что оценка (43) остается справедливой, если предположить, что $x=x(n)=o\left((\ln n)^{1 / 6}\right)$ и остаточный член вида $O\left(1 /(\ln n)^{2}\right)$ заменить на $O\left(\left(1+x^{12}\right) /(\ln n)^{2}\right)$.

Проиллюстрируем точность оценок (23), (41), (43) для одного из рассмотренных выше примеров. Если $n=20$ и $N=3$, то $\gamma=3 / \ln 20 \approx 1,00142$ с точностью до $10^{-5}$ и вычисления по формуле (23) с отбрасыванием остаточного члена порядка $O\left(1 /(\ln n)^{2}\right)$ дают значение 0,27336 (с той же точностью) с относительной погрешностью порядка $-0,005$. При этом $x=(3-\ln 20) / \sqrt{\ln 20} \approx 0,00247$, и вычисления по формулам (41), (43) дают значения 0,27328 и 0,27463 с относительными погрешностями, равными соответственно $-0,006$ и $-0,0007$.

Теорема 3. Пусть при условиях теоремы $2 \gamma_{1}<1$. Тогда равномерно относительно $\gamma \in\left[\gamma_{0}, \gamma_{1}\right]$

$$
\begin{aligned}
& \mathbf{P}\left\{v_{n} \leqslant N\right\}=\frac{(\ln n)^{N}}{N ! n \Gamma(\gamma)}(1-\gamma)^{-1} \\
& \quad \times\left(1+\frac{\gamma\left(\psi^{\prime}(\gamma)-(\psi(\gamma))^{2}+2 \psi(\gamma)(1-\gamma)^{-1}-2(1-\gamma)^{-2}\right)}{2 \ln n}+O\left(1 /(\ln n)^{2}\right)\right) .
\end{aligned}
$$

Доказательство. Согласно (24)

$$
\begin{aligned}
\mathbf{P}\left\{v_{n} \leqslant N\right\} & =\sum_{m=1}^{N} \mathbf{P}\left\{v_{n}=m\right\} \\
& =\frac{1}{2 \pi i n !} \oint \frac{(z+1) \ldots(z+n-1)}{z^{N}(1-z)} d z-\frac{1}{2 \pi i n !} \oint \frac{(z+1) \ldots(z+n-1)}{1-z} d z,
\end{aligned}
$$

где интегрирование в обоих случаях осуществляется по окружности $|z|=z_{0}$, пробегаемой в положительном направлении. Так как $\gamma_{1}<1$, существует постоянная $z_{1} \in(0,1)$ такая, что $z_{0}=z_{0}(n, N) \leqslant z_{1}$ при всех достаточно больших значениях $n, N$ и $\gamma \in\left[\gamma_{0}, \gamma_{1}\right]$. Но тогда согласно интегральной теореме Коши второй интеграл в последнем равенстве для таких значений $n, N$ равен нулю. Применяя к оценке первого интеграла метод перевала и рассуждая так же, как при доказательстве теоремы 2 , получаем, что

$$
\mathbf{P}\left\{v_{n} \leqslant N\right\}=\left(1-z_{0}\right)^{-1} \frac{z_{0}^{-1} \exp \left(N \omega\left(z_{0}\right)\right)}{n ! \sqrt{2 \pi N \omega^{\prime \prime}\left(z_{0}\right)}}\left(1+B_{1} / N+O\left(N^{-2}\right)\right),
$$


где аналогично (29)

$$
\begin{aligned}
B_{1}=- & \frac{1}{4 \varphi_{1}\left(z_{0}\right) \omega^{\prime \prime}\left(z_{0}\right)}\left(2 \varphi_{1}^{\prime \prime}\left(z_{0}\right)-\left(2 \omega^{\prime \prime \prime}\left(z_{0}\right) \varphi_{1}^{\prime}\left(z_{0}\right)\right) /\left(\omega^{\prime \prime}\left(z_{0}\right)\right)\right. \\
& \left.+\varphi_{1}\left(z_{0}\right)\left(\left(5\left(\omega^{\prime \prime \prime}\left(z_{0}\right)\right)^{2}\right) /\left(6\left(\omega^{\prime \prime}\left(z_{0}\right)\right)^{2}\right)-\left(\omega^{(4)}\left(z_{0}\right)\right) /\left(2 \omega^{\prime \prime}\left(z_{0}\right)\right)\right)\right), \\
\varphi_{1}(z)= & z^{-1}(1-z)^{-1} .
\end{aligned}
$$

Подставляя в (46) значения функщии $\varphi_{1}\left(z_{0}\right)$ и ее производных $\varphi_{1}^{\prime}\left(z_{0}\right)$ и $\varphi_{1}^{\prime \prime}\left(z_{0}\right)$, а также найденные ранее значения $\omega^{(k)}\left(z_{0}\right), 2 \leqslant k \leqslant 4$, и учитывая (33), после элементарных преобразований приходим к равенству

$$
B_{1}=-1 / 12-\gamma^{2}(1-\gamma)^{-2}+O(1 / \ln n) .
$$

Теперь оценка (44) следует из соотношений (37), (39), (45), (47), поскольку согласно (33)

$$
\left(1-z_{0}\right)^{-1}=(1-\gamma)^{-1}\left(1+\frac{\gamma \psi(\gamma)}{(1-\gamma) \ln n}+O\left(1 /(\ln n)^{2}\right)\right) \text {. }
$$

Теорема 3 доказана.

Теорема 4. Пусть при условиях теоремы $2 \gamma_{0}>1$. Тогда равномерно отнссительно $\gamma \in\left[\gamma_{0}, \gamma_{1}\right]$

$$
\begin{aligned}
& \mathbf{P}\left\{v_{n} \geqslant N\right\}=\frac{(\ln n)^{N}}{N ! n \Gamma(\gamma)} \gamma(\gamma-1)^{-1} \\
& \quad \times\left(1+\frac{\gamma\left(\psi^{\prime}(\gamma)-(\psi(\gamma))^{2}\right)-2 \psi(\gamma)(\gamma-1)^{-1}-2(\gamma-1)^{-2}}{2 \ln n}+O\left(1 /(\ln n)^{2}\right)\right) .
\end{aligned}
$$

Доказательство. Теорема 4 доказывается аналогично теореме 3. Следует учесть, что

$$
P\left\{v_{n} \geqslant N\right\}=\sum_{m=N}^{\infty} P\left\{v_{n}=m\right\}=\frac{1}{2 \pi i n !} \oint_{|z|=z_{(1)}>1} \frac{(z+1) \ldots(z+n-1)}{(z-1) z^{N-1}} d z,
$$

причем из условия $\gamma_{0}>1$ вытекает, что сушествует постоянная $z_{2}>1$, такая, что $z_{0}=z_{0}(n, N) \geqslant z_{2}$ при всех достаточно больших значениях $n, N$. Применяя к оценке полученного интеграла метод перевала, заменяя в предыдушем доказательстве функцию $\varphi_{1}(z)=z^{-1}(1-z)^{-1}$ на $\varphi_{2}(z)=(z-1)^{-1}$ и замечая, что согласно (33)

$$
z_{0}\left(z_{0}-1\right)^{-1}=\gamma(\gamma-1)^{-1}\left(1-\frac{\psi(\gamma)}{(\gamma-1) \ln n}+O\left(1 /(\ln n)^{2}\right)\right),
$$

приходим к равенству

$$
\begin{aligned}
\mathbf{P}\left\{\nu_{0}\right. & \geqslant N\}=\frac{(\ln n)^{N}}{N ! n \Gamma(\gamma)} \gamma(\gamma-1)^{-1} \\
& \times\left(1+\frac{\gamma\left(\psi^{\prime}(\gamma)-(\psi(\gamma))^{2}\right)-2 \psi(\gamma)(\gamma-1)^{-1}+\left(2 B_{2}+1 / 6\right) \gamma^{-1}}{2 \ln n}+O\left(1 /(\ln n)^{2}\right)\right),
\end{aligned}
$$

где значение $B_{2}$ вычисляется аналогично (46) с заменой $\varphi_{1}\left(z_{0}\right)$ на $\varphi_{2}\left(z_{0}\right)$ и производных $\varphi_{1}^{\prime}\left(z_{0}\right), \varphi_{1}^{\prime \prime}\left(z_{0}\right)$ на $\varphi_{2}^{\prime}\left(z_{0}\right), \varphi_{2}^{\prime \prime}\left(z_{0}\right)$. В результате получаем, что

$$
B_{2}=-1 / 12-\gamma(\gamma-1)^{-2}+O(1 / \ln n) .
$$

Подставляя (50) в (49), получаем (48). Теорема 4 доказана. 


\section{Список литературы}

1. Риордан Дж., Введение в комбинаторный анализ. ИЛ, Москва, 1963.

2. Колчин В. Ф., Случайные графы. Наука, Москва, 2000.

3. Good I. J., An asymptotic formula for the differences of the powers at zero. Ann. Math. Statist. (1961) 32, №1, 249-256.

4. Тимашёв А. Н., Об асимптотических разложениях для чисел Стирлинга 1-го и 2-го рода. Дискретная математика (1998) 10, №3, 148-159.

5. Гончаров В. Л., Из области комбинаторики. Изв. АН СССР, сер. матем. (1944) 8, №1, 3-48.

6. Колчин В. Ф., Севастьянов Б. А., Чистяков В. П., Случайные размещения. Наука, Москва, 1976.

7. Петров В. В., Суммы независимых случайных величин. Наука, Москва, 1972.

8. Сачков В. Н., Комбинаторные методы дискретной математики. Наука, Москва, 1977.

9. Тимашёв А. Н., Об асимптотических разложениях в локальных предельных теоремах в равновероятных схемах размещения частиц по различным ячейкам. Дискретная математика (2000) 12, №1, 60-69.

10. Евграфов М. А., Асииптотические оченки и целые функции. Физматлит, Москва, 1962.

11. Олвер Ф., Введение в асимптотические методы и спечиальные функиии. Наука, Москва, 1978.

12. Бейтмен Г., Эрдейи А., Высшие трансиендентные функиии. Гипергеометрическая функция. Функиии Лежандра. Наука, Москва, 1973.

13. Павлов Ю. Л., О случайных отображениях с ограничениями на число циклов. Tруды Матем. ин-та им. В. А. Стеклова (1986) 177, 131-142.

14. Павлов А. И., Локальные теоремы для числа компонент в случайных подстановках и отображениях. Теория вероятностей и ее применения (1988) 33, №1, 196-200.

15. Волынец Л. М., Оценка скорости сходимости к предельному распределению для числа циклов в случайной подстановке. В кн.: Вероятностные задачи дискретной математики. МИЭМ, Москва, 1987, с. $40-46$.

16. Тимашёв А. Н., Случайные разбиения множеств с известным числом блоков. Дискретная математика (2003) 15, №2, 138-148. 